Trauma Berufskrankh 2015 • 17:244-249 DOI 10.1007/s10039-015-0093-6

Online publiziert: 19. Oktober 2015

(c) Die Autor(en) 2015. Dieser Artikel ist auf Springerlink.com mit Open Access verfügbar

CrossMark

B. Kusma ${ }^{1} \cdot$ J.-J. Glaesener' $\cdot$ S. Brandenburg ${ }^{3} \cdot$ A. Pietsch ${ }^{2} \cdot$ K. Fischer ${ }^{4} \cdot$ J. Schmidt ${ }^{5}$. S. Behl-Schön ${ }^{3} \cdot$ U. Pohrt ${ }^{1}$

${ }^{1}$ Berufsgenossenschaft für Gesundheitsdienst und Wohlfahrtspflege (BGW), Grundlagen der Prävention und Rehabilitation, Berufsdermatologie und Interventionsstrategien, Berlin, Deutschland

2 Berufsgenossenschaftliches Unfallkrankenhaus, Hamburg, Deutschland

${ }^{3}$ Berufsgenossenschaft für Gesundheitsdienst und Wohlfahrtspflege (BGW), Hamburg, Deutschland

${ }^{4}$ BG Kliniken Bergmannstrost, Halle, Deutschland

${ }^{5}$ BG Nordsee Reha-Klinik, Sankt Peter Ording, Deutschland

\title{
Der Pflege das Kreuz stärken - Individualprävention „Rücken“ bei der Berufsgenossenschaft für Gesundheitsdienst und Wohlfahrtspflege
}

\section{Das Konzept des Rückenkollegs}

Beschäftigte in der Pflege haben aufgrund berufstypischer, wirbelsäulenbelastender Tätigkeiten ein erhöhtes Risiko für bandscheibenbedingte Erkrankungen der Lendenwirbelsäule (BK 2108). Im Jahr 2014 ging bei der Berufsgenossenschaft Gesundheitsdienst und Wohlfahrtspflege (BGW) 3065-mal die Meldung auf den Verdacht der BK 2108 ein. Sofern bereits einschlägige Symptome vorliegen, der Arbeitsplatz durch entsprechende Belastungen charakterisiert ist und die Betroffenen noch an diesem tätig sind, bietet die BGW ihren Versicherten die 3-wöchige Präventionsmaßnahme BGW-Rückenkolleg an.

\section{Zielsetzung des BGW-Rückenkollegs}

Zielstellung des Rückenkollegs ist es, Versicherte mit Lendenwirbelsäulenerkrankungen beim Verbleib am Arbeitsplatz zu unterstützen. Durch die Vermittlung eines mehrdimensionalen, biopsychosozialen Krankheitsverständnisses wird die individuelle Handlungskompetenz bei der Gesunderhaltung des Rückens gestärkt und die Entwicklung eines grundlegend positiven Funktionsbildes des Rü- ckens unterstützt. Die Schulung zielt darauf ab, die persönliche Schmerzreaktion zu reflektieren und verschiedene Bewältigungsmöglichkeiten aufzuzeigen. Durch das Erlernen von wirbelsäulenentlastenden Arbeitstechniken, die Förderung eines adäquaten Bewegungsverhaltens im Alltag und ein intensives Muskelaufbautraining wird die Gesamtbelastbarkeit der Versicherten verbessert.

\section{Zielgruppe und \\ Zugangsvoraussetzungen}

Die Maßnahme wird den Versicherten auf der Grundlage des $₫ 3$ der Berufskrankheitenverordnung (BKV; [5]) zur Prävention einer drohenden Berufskrankheit BK 2108 angeboten. Diese wird wie folgt definiert:

Bandscheibenbedingte Erkrankungen der Lendenwirbelsäule durch langjähriges Heben oder Tragen schwerer Lasten oder durch langjährige Tätigkeiten in extremer Rumpfbeugehaltung, die zur Unterlassung aller Tätigkeiten gezwungen haben, die für die Entstehung, die Verschlimmerung oder das Wiederaufleben der Krankheit ursächlich waren oder sein können
Bei der BGW sind insbesondere Pflegekräfte in der stationären und ambulanten Alten- und Krankenpflege betroffen. Voraussetzung für die Teilnahme am Rückenkolleg ist neben einer gefährdenden beruflichen Tätigkeit und einem Krankheitsbild entsprechend der BK 2108 eine ausreichende Belastbarkeit für die Trainingseinheiten.

\section{Theoretische Fundierung}

Das Rückenkolleg ist den Leitlinien zur Behandlung von chronischem Rückenschmerz sowie aktuellen Behandlungsempfehlungen und -konzepten entsprechend bewegungsbezogen und biopsychosozial ausgerichtet $[3,20]$.

Rückenschmerzen werden hier als ein multifaktorielles Geschehen eingestuft [15]. So stehen somatische Beschwerden zwar am Anfang der Kausalkette, verlieren aber aufgrund multipler Beeinflussung durch psychosoziale Faktoren wie subjektive Theorien der Betroffenen, das individuelle Krankheitsverhalten und Einflüsse der Umwelt zunehmend an Bedeutung [21]. Die Folgen können psychische Beeinträchtigungen, Verhaltensänderungen (z. B. Schon- und Ver- 
meidungsverhalten), dadurch begünstigte körperliche Dekonditionierung (u. a. der Rückenmuskulatur; [17]), inadäquate Krankheitsbewältigung und soziale Auswirkungen sein. Indem sich das Rückenkolleg am Teilnehmer orientiert und individuelle Lösungsstrategien für rückengerechte Arbeitsweisen angeboten werden, reduzieren sich angstvermeidende Bewegungskognitionen. Mit der Vermittlung von Wissen wird der Schmerz entdramatisiert, und die Kompetenzen zum aktiven Umgang mit den Beschwerden werden erhöht.

Durch Trainingsmaßnahmen zur Verbesserung der Gesamtbelastung im Beruf und Alltag sowie Entspannungsverfahren zur Stress- bzw. Schmerzbewältigung erleben die Teilnehmer Belastungsverträglichkeit in positiver Form [13].

Diese Kombination aus Edukation, Verhaltens- und Bewegungstherapie innerhalb eines biopsychosozialen, multidisziplinären Interventionsansatzes ist bei chronischen Rückenschmerzpatienten grundsätzlich geeignet, Schmerzen zu reduzieren und eine Funktionsverbesserung zu erreichen [9]. Das Rückenkolleg erweitert diesen Ansatz durch den Bezug auf den individuellen Arbeitsplatz der Teilnehmer, da Studien gezeigt haben, dass in der Therapie chronischer Rückenschmerzen Interventionsformen, die arbeitsplatzspezifische Merkmale berücksichtigen, wirksam sind $[7,10,16]$.

Das Rückenkolleg orientiert sich zudem an dem Konzept der Salutogenese, d. h., es wird nach Schutzfaktoren gesucht, die einer Person helfen, trotz pathogener Bedingungen gesund zu bleiben bzw. sich bei Erkrankungen/Symptomen gesund zu fühlen [13]. Nach Antonovsky [1] bilden die Zustände "gesund“ und „krank“ ein Kontinuum mit fließendem Übergang. Bezogen auf die Rückengesundheit heißt das, dass der Mensch ständig gefordert ist, diese zu erlangen oder zu erhalten [13]. Wo sich eine Person auf dem Kontinuum lokalisiert, hängt von der individuellen Fähigkeit ab, Spannungszustände auszugleichen. Diese entstehen durch die Interaktion von Stressoren, Effizienz der Spannungsbewältigung, generalisierten Widerstandsquellen und dem Kohärenzsinn. Von der Art der Spannungsbewältigung hängt es ab, ob Stressoren in pa- thologischer, neutraler oder gesundheitsfördernder Richtung auf dem Gesundheits-Krankheits-Kontinuum wirken [2]. Der Ansatz des Rückenkollegs, berufsbedingten Rückenbeschwerden durch einen verbesserten Umgang mit Belastungen im Arbeitsalltag entgegenzuwirken, steht für eine gesundheitsförderliche, salutogene Herangehensweise.

Beide Ansätze werden in einem multimodalen Kursprogramm umgesetzt, das sowohl verhaltens- als auch verhältnispräventive Aspekte beinhaltet [12] und die Eigenverantwortung der Teilnehmer und die Fähigkeit, selbstwirksam zu handeln, fördert.

\section{Konzept des Rückenkollegs}

Das Rückenkolleg integriert orthopädische, sportmedizinische und trainingswissenschaftliche, physio- und ergotherapeutische sowie psychologische $\mathrm{Me}$ thoden. Eine multiprofessionelle Diagnostik gewährleistet ein individuell abgestimmtes, patientenspezifisches Vorgehen. Während der 3-wöchigen Präventionsmaßnahme erlernen die Teilnehmer in den täglichen Trainings- und Schulungseinheiten, wie sie mit den berufsbedingten Belastungen des Rückens und Bewegungsapparates besser umgehen und arbeitsspezifische Abläufe ökonomisieren können. Das Rückenkolleg umfasst folgende Kernelemente:

- Vermittlung von Wissen und Fertigkeiten zur Förderung der Funktionsfähigkeit,

- medizinische Trainingstherapie zur Kräftigung der Rumpfmuskulatur und Verbesserung der körperlichen Grundstabilität, Körperwahrnehmung, Ausdauerleistung und Koordination sowie Physiotherapie,

- berufsspezifisches Üben unter Anwendung ergonomischer Grundprinzipien in unterschiedlichen Patiententransfersituationen sowie

- psychologisches Gesundheitstraining zum Erwerb und Ausbau verschiedener Strategien für einen verbesserten Umgang mit Schmerzen, Stress und psychischen Belastungssituationen.

\section{Vermittlung von Wissen und Fertigkeiten}

\section{Arztvortrag}

Durch die Vermittlung von rückenbezogenen Informationen lassen sich die mit Rückenschmerz zusammenhängenden Kognitionen und das entsprechende Verhalten beeinflussen [19]. Dementsprechend werden in einem ärztlich geleiteten Modul Kenntnisse über die Anatomie, Physiologie und Biomechanik der Wirbelsäule sowie über die pathophysiologischen Grundlagen von Wirbelsäulenerkrankungen vermittelt und die Therapieoptionen und Präventionsmöglichkeiten vorgestellt. Wesentlich ist dabei die Botschaft, dass wahrgenommene Schmerzen nicht dem Ausmaß körperlicher Schädigung entsprechen müssen, die Wirbelsäule ein stabiles und muskulär gut zu sicherndes System darstellt und dass die Beibehaltung von Aktivität der beste Weg zur Vermeidung von Rückenschmerzen ist.

\section{Schulung zu Hilfsmitteln und deren Einsatz}

Das Maß, der in der Pflege auftretenden Belastungen der Wirbelsäule, kann durch eine rückengerechte Haltung und durch rückengerechte Bewegungsabläufe in Verbindung mit der Nutzung der Ressourcen der Patienten reduziert werden. Ein wesentlicher Faktor für die Minderung der Druckbelastung ist die Kombination der optimierten Arbeitsweise mit der Verwendung von Hilfsmitteln [11]. In einem entsprechenden Modul werden die Teilnehmer von einem Rehabilitationsfachberater über die Möglichkeiten mit kleinen und großen Hilfsmitteln, rückengerecht zu arbeiten, informiert. Vorgestellt werden u. a. Liftersysteme, Aufstehhilfen, Rutsch- und Rollbretter sowie Drehscheiben. Die Schulung hat einen umfangreichen praktischen Teil, um einen möglichst hohen Lernerfolg zu sichern.

\section{Ernährungsberatung}

Eine gesunde Ernährung wirkt sich entscheidend auf die Gesundheit und das Wohlbefinden des Einzelnen aus und kann Beeinträchtigungen und Krankheiten behandlungsunterstützend positiv beeinflussen. Das Körpergewicht bzw. der 
Body-Mass-Index scheinen zudem einen Einfluss auf die Schmerzempfindung zu haben. In einer Risikogruppenanalyse von Ochsmann et al. [18] gaben übergewichtige und adipöse Personen im Vergleich zu Normalgewichtigen eher starke Rückenschmerzen an. In der Ernährungsberatung lernen die Versicherten gesunde und ausgewogene Ernährung kennen. Die Schulung ist teilnehmerzentriert und handlungsorientiert, um Verhaltensänderungen zu initiieren. Neben der Information steht insbesondere die Motivierung der Teilnehmer im Sinne der Handlungsaktivierung und Reflexion des Ernährungsverhaltens im Fokus. Im theoretischen Teil wird über gesunde Ernährung informiert (Dauer 60-90 min), im praktischen Teil (Dauer 90-120 min) wird ein Koch- bzw. Einkaufstraining durchgeführt.

\section{BGW-Schulung}

Das Rückenkolleg ist ein Angebot der BGW zur sekundären Individualprävention ( $\$ 3$ Berufskrankheitenverordnung, BKV). Die Teilnehmer werden von einem Fachberater über die Aufgaben und Leistungen der gesetzlichen Unfallversicherung bei einer Berufskrankheit Nr. 2108, zu Angeboten der BGW und Zielen des Rückenkollegs informiert sowie zu ihrer individuellen Situation in diesem Zusammenhang beraten.

\section{Therapeutischer Bereich}

Die therapeutische Arbeit ist ausgerichtet auf:

- die Behandlung und Schulung eines besseren Körperbewusstseins,

- die Verbesserung der Gesamtbelastbarkeit im Alltag und im Beruf durch aktive Maßnahmen,

- die Übernahme von Eigenverantwortung für das körperliche Wohlbefinden und

- die Vermittlung einfacher und alternativer Therapiemethoden zur Selbstbehandlung.

Passive Maßnahmen zur Schmerzreduktion und Regeneration begleiten die therapeutische Arbeit.

Trauma Berufskrankh 2015 • 17:244-249 DOI 10.1007/s10039-015-0093-6

(c) Die Autor(en) 2015. Dieser Artikel ist auf Springerlink.com mit Open Access verfügbar

B. Kusma · J.-J. Glaesener · S. Brandenburg · A. Pietsch · K. Fischer · J. Schmidt · S. Behl-Schön • U. Pohrt

\section{Der Pflege das Kreuz stärken - Individualprävention „Rücken“ bei der Berufsgenossenschaft für Gesundheitsdienst und Wohlfahrtspflege. Das Konzept des Rückenkollegs}

\section{Zusammenfassung}

Die Berufsgenossenschaft für Gesundheitsdienst und Wohlfahrtspflege (BGW) bietet für Beschäftigte in Gesundheits- und Pflegeberufen, bei denen eine bandscheibenbedingte Erkrankung der Lendenwirbelsäule (LWS) entsprechend der Berufskrankheiten (BK)-Ziffer 2108 droht, das „Rückenkolleg“ an. Das BGW-Rückenkolleg ist eine 3-wöchige Präventionsmaßnahme mit multimodaler Ausrichtung unter besonderen berufsspezifischen Aspekten. Übergeordnetes Ziel des Angebotes ist es, Betroffene mit LWS-Erkrankun- gen beim Verbleib am Arbeitsplatz zu unterstützen und ein frühzeitiges Ausscheiden aus dem Beruf zu verhindern. Dieser Artikel beschreibt das Konzept, die theoretische Fundierung sowie die verschiedenen präventiven und therapeutischen Maßnahmen des Rückenkollegs.

Schlüsselwörter

Pflegeberufe - Berufskrankheit .

Rückenschmerzen · Prävention ·

Lendenwirbelsäule

\section{To back nurses up: the individual back problem prevention program by the Institution for Statutory Accident Insurance in Health and Welfare Services. The concept of the „back college"}

\section{Abstract}

The Institution for Statutory Accident Insurance in Health and Welfare Services (BGW) offers the "back college" to employees in healthcare and nursing professions with an imminent occupational disease related to vertebral discs of the lumbar spine (OD 2108). This 3-week inpatient treatment course is a preventive measure based on a multimodal concept with job-related aspects. The overall objectives of the course are to support those affected to remain in their jobs and to pre- vent premature discontinuation of the occupation. This article describes the concept and theoretical foundations as well as the various preventive and therapeutic measures provided by the "back college".

\section{Keywords} Nursing personnel - Low back pain . Occupational disease - Lumbar spine . Prevention

\section{Sporttherapie: medizinische Trainingstherapie}

In der medizinischen Trainingstherapie lernen die Versicherten grundlegende Trainingsprinzipien kennen und umzusetzen. Jeder Versicherte erhält einen individuellen Trainingsplan, der die Ergebnisse der funktionsdiagnostischen Testung zu Beginn des Rückenkollegs und das momentane Beschwerdebild berücksichtigt und wöchentlich entsprechend des Leistungsvermögens und Trainingszustandes angepasst wird.

Die regulären Trainingseinheiten von mehreren Stunden täglich beinhalten Übungen zur Stabilisation und Gewichtsverlagerung (z. B. an den Seilzügen, auf dem Wackelbrett), Koordinationstraining zum Aufbau der Haltung und des Fuß- gewölbes, Bodenübungen auf der Matte und an medizinischen Trainingsgeräten zur Kräftigung der tiefen Bauch- und Rückenmuskulatur sowie Beckenbodentraining (•Abb. 1).

Weiterhin Schwerpunkte sind Detonisierungsübungen für den Schultergürtel sowie das intensive Trainieren von Squats, um die Gesäß- und Beinmuskulatur zu kräftigen - eine wesentliche Voraussetzung für die Entlastung des Rückens durch einen vermehrten Beineinsatz. Die einzelnen Trainingselemente sollen die Teilnehmer befähigen, rückenschonende ergonomische Bewegungsabläufe im berufsspezifischen Üben (BSÜ) und später auch im Beruf und Alltag umzusetzen. Zur Optimierung der Gesamtbelastbarkeit und Verbesserung der Aus- 


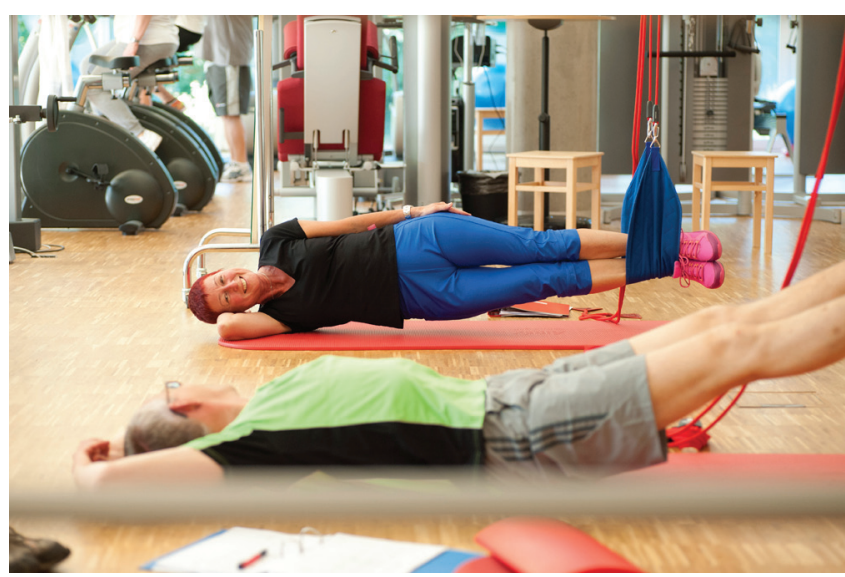

Abb. 1 A Medizinische Trainingstherapie

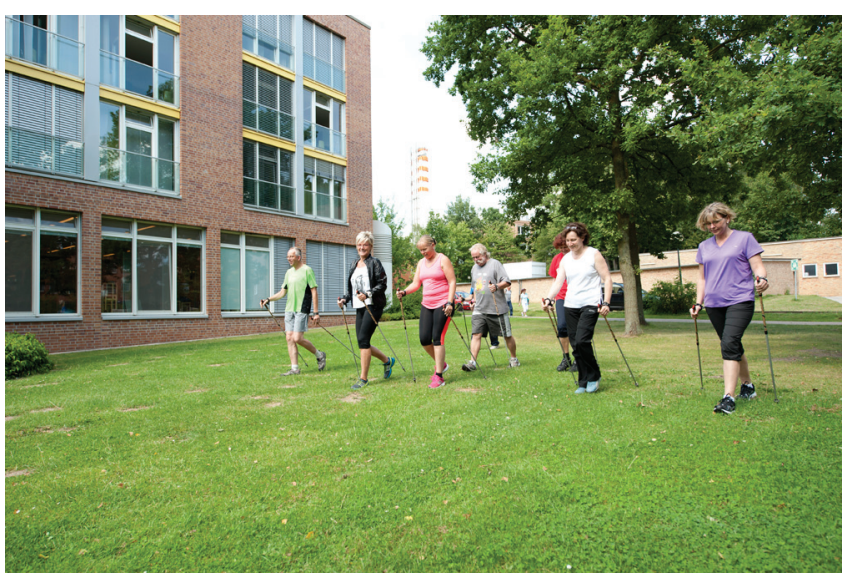

Abb. 2 ム Nordic Walking in der Gruppe dauerfähigkeit ist auch das Kardiotraining, z. B. auf dem Cross-Trainer, Fahrradergometer oder Laufband, fester Bestandteil der medizinischen Trainingstherapie. Dazu gehören außerdem Nordic Walking (• Abb. 2) und Aquagymnastik/Schwimmen.

\section{Physiotherapie}

Die konkreten physiotherapeutischen Einzelbehandlungen ergeben sich aus der Eingangsbefundaufnahme. Der individuelle Behandlungsplan setzt sich bedarfsorientiert aus Behandlungskonzepten der manuellen Therapie, propriozeptiven neuromuskulären Fazilitation, Krankengymnastik auf neurophysiologischer Basis und Traktionstherapie zusammen. Mit der Behandlung werden Körperwahrnehmung und Körperaufrichtung verbessert, die Wirbelsäulenbeweglichkeit erhöht und Schmerzen verringert. Außerdem fördert sie die Anbahnung und Ansteuerung benötigter Muskulatur und verhilft zu einer segmentalen Stabilisation und einem Ausgleich muskulärer Dysbalancen. Der Therapeut geht dabei auf individuelle Bewegungsabläufe aus dem berufsspezifischen Üben ein und vertieft sie. Durch die Übungen verbessert sich die Stabilität bei berufsspezifischen und alltagsrelevanten Bewegungen - besonders um körpergerechtes Bewegen von Lasten zu ermöglichen. Ziel ist die eigenverantwortliche Durchführung eines persönlichen Übungsprogramms, damit der Teilnehmer das Programm später zu Hause fortführen kann.

Begleitende physikalische Therapiemaßnahmen regen den Stoffwechsel an, reduzieren Schmerzen, entspannen die Muskulatur und unterstützen so die aktiven Kursmodule.

\section{Entspannungstherapie}

Hier lernen die Versicherten verschiedene anerkannte Methoden und bewährte Praxisbeispiele zur Entspannung kennen. Dazu gehören Atemübungen, Dehnungen, Fantasiereisen mit Aspekten aus dem autogenen Training, Qi Gong, Yoga und progressive Muskelrelaxation. Durch diese Methodenvielfalt können die Teilnehmer eigene Vorlieben entdecken, da nicht jedes Verfahren gleichermaßen für alle geeignet ist. Mithilfe von Entspannungsverfahren lernen die Versicherten, Körperfunktionen positiv zu beeinflussen, was zu einem veränderten Umgang mit Belastungen führen kann [6]. Die Teilnehmer werden in die Lage versetzt, sich selbst zu beeinflussen und eine positive Wirkung zu erreichen.

\section{Psychologisches Gesundheitstraining}

Mit diesem Modul wird der Tatsache Rechnung getragen, dass vor allem psychische und psychosoziale Faktoren eine besondere Rolle bei der Chronifizierung von Rückenschmerzen spielen [8, 14]. Das psychologische Gesundheitstraining dient zum einem der Wissensvermittlung, zum anderen werden die Teilnehmer da$\mathrm{zu}$ angehalten, individuelle Stressoren und ihre Bewältigungsstrategien kritisch zu bewerten. Sie lernen alternative, geeignetere Reaktions- und Umgangsformen für belastendende Situationen und auf- tretende Schmerzen kennen und trainieren diese.

Das psychologische Gesundheitstraining umfasst 5 Einheiten von jeweils $90 \mathrm{~min}$ :

- Stress/Belastung,

- Schmerz,

- Aktivitätsregulation,

- Selbstwahrnehmung,

- Transfersicherung.

\section{Berufsspezifisches Üben}

Das BSÜ ist ein wesentlicher Bestandteil des Rückenkollegs. Es dient dem Erlernen ergonomischer Grundprinzipien mit dem Ziel, das Heben und Tragen auf ein Minimum zu reduzieren und die Patienten durch Ausnutzen individueller Ressourcen aktiv mit einzubeziehen.

Nach einer theoretischen Einführung, bei der die wichtigsten Elemente für das ergonomische Arbeiten zusammengetragen und erläutert werden, ist das BSÜ vorrangig praktisch ausgelegt. Die Teilnehmer lernen, wie sie mithilfe von Bewegungselementen aus dem Bobath- und Kinästhetikkonzept unterschiedliche $\mathrm{Pa}$ tiententransfers ergonomisch bewältigen (• Abb. 3) Darüber hinaus zielt das BSÜ auf eine Verbesserung der im Berufsalltag notwendigen Kraft und Ausdauer. Gleichzeitig sollen die Versicherten die individuellen Grenzen ihrer Belastbarkeit rechtzeitig erkennen und respektieren.

Die Teilnehmer werden intensiv im Umgang mit Hilfsmitteln, in der Körperwahrnehmung, dem Gleichgewicht und der Koordination bei Gewichtsverlagerungen zum Schieben und Ziehen von 


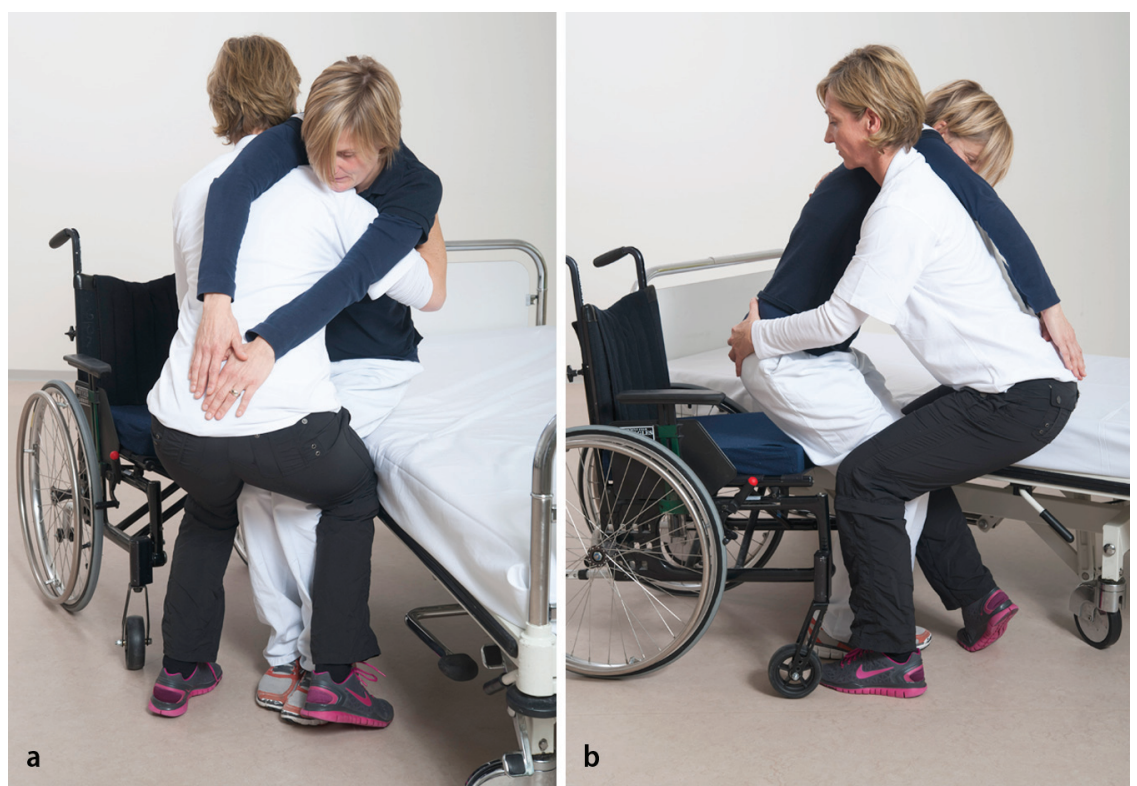

Abb. $3 \Delta$ a, b Anwendung einer rückenschonenden Technik beim Patiententransfer

Lasten im Arbeitsprozess geschult. Beispiele aus dem Arbeitsalltag verdeutlichen theoretische und praktische Inhalte.

\section{Nachsorge}

Die umfassenden Lebensstiländerungen, wie sie das Rückenkolleg (rückengerechtes Arbeiten, Steigerung der körperlichen Aktivität etc.) anregt, können in den 3 Wochen lediglich initiiert werden. Mithilfe von Nachsorgemaßnahmen besteht die Möglichkeit, Lebensstiländerungen zu stabilisieren und die Nachhaltigkeit der medizinischen Rehabilitation zu erhöhen [4].

Im Anschluss an das Rückenkolleg bietet die BGW daher ihren Versicherten verschiedene Maßnahmen an, um den Transfer zu sichern.

\section{Arbeitsplatzbegleitung}

Etwa 12 Wochen nach dem Rückenkolleg findet eine Arbeitsplatzbegleitung (APB) statt. Eine Trainerin oder ein Trainer der BGW begleitet den Versicherten durch seinen Arbeitstag - in der Regel über 2 Schichten.

Ziel ist, die im Rückenkolleg erlernten Inhalte und Techniken am individuellen Arbeitsplatz umzusetzen und in die Alltagsroutine zu überführen sowie die Versicherten zur Aufrechterhaltung rücken- gerechter Arbeits- und Verhaltensweisen im Berufsalltag zu motivieren.

Die Inhalte des Rückenkollegs, besonders aus dem BSÜ, werden wiederholt und positiv unterstützt, um das Handeln des Versicherten zu festigen.

Der Verlauf der Arbeitsplatzbegleitung wird dokumentiert und bildet die Grundlage des Abschlussgesprächs. Anhand der beobachteten Arbeitsabläufe und Situationen empfehlen die Arbeitsplatzbegleiter konkrete Maßnahmen zur Etablierung rückengerechter Arbeitsbedingungen.

\section{Refresher-Kurs}

Die Teilnehmer können 1 bis 1 1/2 Jahre nach der Teilnahme am Rückenkolleg einen 5-tägigen Refresher-Kurs absolvieren, wenn sie weiterhin einen gefährdenden Beruf ausüben und aktiv und motiviert am Rückenkolleg teilgenommen haben. Durch das Refresher-Konzept werden die Inhalte des Rückenkollegs aufgefrischt und Probleme aufgegriffen, die nach dem Rückenkolleg im beruflichen Alltag aufgetreten sind.

\section{Fazit für die Praxis}

- Das Rückenkolleg ist eine Individualpräventionsmaßnahme für Pflegekräfte mit drohender BK 2108, die sich an aktuellen Erkenntnissen zur Therapie/Rehabilitation von Rückenschmerzen orientiert.

- Ziel ist der Erhalt der Arbeitsfähigkeit im Beruf.

- Die Effektivität dieser Maßnahme wurde bisher nur retrospektiv nachgewiesen. Eine entsprechende Studie mit prospektivem Ansatz wird derzeit durchgeführt. Erste Ergebnisse bestätigen die Wirksamkeit.

\section{Korrespondenzadresse}

\section{B. Kusma}

Berufsgenossenschaft für Gesundheitsdienst und Wohlfahrtspflege (BGW), Grundlagen der Prävention und Rehabilitation,

Berufsdermatologie und Interventionsstrategien Spichernstraße 2-3, 10777 Berlin

Bianca.kusma@bgw-online.de

\section{Einhaltung ethischer Richtlinien}

Interessenkonflikt. B. Kusma, J.-J. Glaesener, S. Brandenburg, K. Fischer, J. Schmidt, S. Behl-Schön und U. Pohrt geben an, dass kein Interessenkonflikt besteht. A. Pietsch ist mit der Durchführung des Rückenkollegs beauftragt.

Dieser Beitrag beinhaltet keine Studien an Menschen oder Tieren.

Alle Patienten, die über Bildmaterial oder anderweitige Angaben innerhalb des Manuskripts zu identifizieren sind, haben hierzu ihre schriftliche Einwilligung gegeben.

Open Access Dieser Artikel unterliegt den Bedingungen der Creative Commons Attribution License. Dadurch sind die Nutzung, Verteilung und Reproduktion erlaubt, sofern der/die Originalautor/en und die Quelle angegeben sind.

\section{Literatur}

1. Antonovsky A (1979) Health, stress and coping: new perspectives on mental and physical well-beeing. Josey Bass, San Francisco

2. Blättner B (2007) Das Modell der Salutogenese. Präv Gesundheitsf 2:67-73

3. Böhle E, Bork H, Brüggemann S et al (2013) Rehabilitation von Rückenschmerzen. Indikationsstellung - Best-Practice-Empfehlungen - Nachsorge. Expertenpanel „Rückenschmerz" der Bertelsmann Stiftung, Gütersloh

4. Deck R, Schramm S, Hüppe A (2012) Begleitete Eigeninitiative nach der Reha („neues Credo") ein Erfolgsmodell? Rehabilitation 51:316-325

5. Deutsche Bundesregierung. Berufskrankheiten-Verordnung vom 31. Oktober 1997 (BGBI. I.S. 2623), zuletzt aktualisiert durch Artikel 1 der Verordnung vom 11. Juni 2009 (BGBII S. 1273). BGBL I: 1273 
6. Gebauer K, Zechner F (2011) Entspannung. In: Flothow A, Kempf H-D, Kuhnt U, Lehmann G (Hrsg) KddR-Manual Neue Rückenschule. Elsevier, München, S 162-176

7. Greitemann B, Dibbelt S, Büschel C (2006) Integriertes Orthopädisch-Psychosomatisches Konzept zur medizinischen Rehabilitation von Patienten mit chronischen Schmerzen des Bewegungsapparates - Langfristige Effekte und Nachhaltigkeit eines multimodalen Programms zur Aktivierung und beruflichen Umorientierung. Z Orthop Ihre Grenzgeb 144:255-266

8. Hasenbring M, Marienfeld G, Kuhlendahl D et al (1994) Risk factors of chronicity in lumbar disk patients: a prospective investigation of biologic, psychologic, and social predictors of therapy outcome. Spine 19:2759-2765

9. Hildebrandt J (2005) Paradigmenwechsel im Umgang mit dem Rückenschmerz - Konsequenzen für bewegungstherapeutische Interventionen. BG Beweg Gesundh 21:151

10. Hildebrandt J, Pfingsten M, Lüder S et al (2003) Göttinger Rücken-Intensiv-Programm (GRIP): Manual. Congress Compact Verlag, Berlin

11. Jäger M, Jordan C, Theilmeier A et al (2014) Analyse der Lumbalbelastung bei manuellen Bewegen von Patienten zur Prävention biomechanischer Überlastungen von Beschäftigten im Gesundheitswesen. In: Nienhaus A (Hrsg) RiRe - Risiken und Ressourcen in Gesundheitsdienst und Wohlfahrtspflege. ecomed Medizin, Landsberg am Lech, S 74-100

12. Kempf H-D, Kuhnt U (2011) Die Neue Rückenschule. In: Flothow A, Kempf H-D, Kuhnt U, Lehmann G (Hrsg) KddR-Manual Neue Rückenschule. Elsevier, München, S 59-62

13. Kempf H-D, Steinau M (2011) Ziele und Inhalte der Neuen Rückenschule. In: Flothow A, Kempf H-D, Kuhnt U, Lehmann G (Hrsg) KddR-Manual Neue Rückenschule. Elsevier, München, S 62-71

14. Klenerman L, Slade PD, Stanley IM et al (1995) The prediction of chronicity in patients with an acute attack of low back pain in a general practice setting. Spine (Phila Pa 1976) 20:478-484

15. Lühmann D (2005) Prävention von Rückenschmerz - Grundlagen und mögliche Interventionsstrategien. BG Beweg Gesundh 21:138-145

16. Möller JU, Morfeld M, Hintze R, Fox M, Höder JH, Krauth C, Koch U (2006) Back to Balance. Ein kognitiv-verhaltentherapeutisches Interventionsprogramm bei chronischen Rückenschmerzen in der stationären Rehabilitation, Bd 64. DRV-Schriften, Frankfurt a. M., S 335-337

17. Moseley GL, Nicholas MK, Hodges PW (2004) A randomized controlled trial of intensive neurophysiology education in chronic low back pain. Clin J Pain 20:324-330

18. Ochsmann $\mathrm{E}$, Rüger $\mathrm{H}$, Letzel $\mathrm{S}$ et al (2008) Präventionsansätze für akute Rückenschmerzen. Risikogruppenanalyse. Trauma Berufskrankh 10:272278

19. Pfeifer K (2004) Expertise zur Prävention von Rückenschmerzen durch bewegungsbezogene Interventionen. Im Auftrag der Bertelsmannstiftung und der Akademie für Manuelle Medizin an der Universität Münster. Otto-von-Guericke Universität Magdeburg, Institut für Sportwissenschaften

20. Pfeifer K (2007) Rückengesundheit. Grundlage und Module zur Planung von Kursen. Deutscher Ärzteverlag, Köln

21. Pfingsten $M$ (2011) Psychologie. In: Flothow $A$, Kempf H-D, Kuhnt U, Lehmann G (Hrsg) KddR-Manual Neue Rückenschule. Elsevier, München, S 3845

\section{Hohe Dunkelziffer bei Gehirn- erschütterungen - Neuer Schnelltest für Breiten- und Schulsport}

Beim Sport kann schon ein vermeintlich harmloser Sturz auf den Kopf oder ein Zusammenprall eine Gehirnerschütterung zur Folge haben. Diese Vorfälle werden jedoch häufig nicht ernst genommen. Wie Lehrer, Eltern und Kinder Hinweise auf eine Gehirnerschütterung erkennen und was im Akutfall zu tun ist, berichteten Experten im Rahmen des DKOU 2015 in Berlin. In Deutschland werden pro Jahr mehr als 40.000 Gehirnerschütterungen diagnostiziert, die Dunkelziffer liegt deutlich höher. "Denn Sportler, vor allem im Schul- und Breitensport, unterschätzen diese Unfälle häufig", warnt Dr. Axel Gänsslen, Arzt am Klinikum Wolfsburg. Wird eine Gehirnerschütterung nicht richtig behandelt, können Spätschäden wie etwa Migräne oder Bewegungsstörungen folgen. Alle Anzeichen einer Gehirnerschütterung sind auf der PocketCard des Fußballweltverbandes FIFA zusammengefasst. Darauf basierend gibt es zudem eine neue App "Schütz Deinen Kopf". „Diese sollte ab sofort auch als Schnelltest am Spielfeldrand eingesetzt werden", fordert Prof. Dr. Michael Nerlich, Kongresspräsident des DKOU 2015 und Direktor der Klinik für Unfallchirurgie am Universitätsklinikum Regensburg. Hier sind unter anderem fünf Fragen aufgelistet, die Trainer oder Teamkollegen dem Betroffenen stellen sollten; beispielsweise, wer das letzte Spiel gewonnen hat. Sobald nur eine der Fragen nicht richtig beantwortet wird, bestätigt das den Verdacht einer Gehirnerschütterung und der Spieler muss umgehend aus dem Spiel genommen werden. Ist die Diagnose Gehirnerschütterung durch einen Arzt gesichert, dauert es mindestens sechs bis zehn Tage, bis sich die Nervenzellen erholt haben. In dieser Zeit sollten äußere Reize wie etwa Musik, Computer oder Lernen ausgeschaltet werden. Zeit und Ruhe sind die wichtigsten Therapiebestandteile.

Eine medikamentöse Behandlung gibt es nicht. „Da die Betroffenen den Schmerz oder die Schwellung nicht wie bei einer Verletzung am Gelenk wahrnehmen, bedarf es häufig viel Überzeugungskraft, um Sportler davon abzuhalten, zu früh wieder aktiv zu werden", sagt Gänsslen. Die Prognose sei aber meist gut: $85 \%$ erholen sich vollständig innerhalb einer Woche. Bleiben die Symptome länger als 3-4 Wochen bestehen, sollte eine neurologische Untersuchung erfolgen.

Quelle: Pressekonferenz DKOU 2015 